\section{MEDIACIONES Y MEDIOS DE COMUNICACIÓN PÚBLICA LOCAL: UNA MIRADA DESDE CUMANAYAGUA}

\section{MEDIATION AND MEDIA COMMUNICATION} LOCAL PUBLIC: A LOOK FROM CUMANAYAGUA

\section{Páginas: Dariel Mena Méndez}

104-119 dmenaAucf.edu.cu

12 de abril de 2015 Facultad de Comunicación de la Universidad de La Habana, UH Xacultad de Comunicación de la Universidad de La Habana, UH-

Aceptado

04 de mayo de 2015

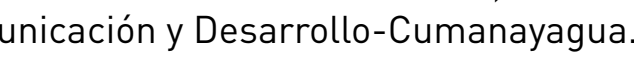

El articulo deriva del proyecto "Estrategias de Comunicación para el Desarrollo Social de Cumanayagua", auspiciado por la UNESCO y financiado por el Programa Internacional para el Desarrollo de la Comunicacion PIDC-Paris, Francia.

\section{Resumen}

Los estudios de mediaciones en Latinoamérica han cobrado mayores auges en las últimas décadas, a partir de las reflexiones analíticas en torno a los medios de comunicación pública comunitarios y locales. Su abordaje se ha caracterizado por un matiz pluridisciplinar, con la participación de diversas Ciencias Sociales como la Sociología, la Psicología y la Comunicología. En investigación, se presentan algunos apuntes y reflexiones en torno a las mediaciones desde la producción de comunicación pública local del municipio Cumanayagua y su efecto en los procesos de socialización y comportamiento humano en los espacios públicos. Para lo cual, acudimos a los presupuestos de Manuel M Serrano (1977, 1985, 2008), Jesús Martín

Barbero $(1987,1995)$ y Guillermo Orozco (1991)

Palabras clave Mediaciones, medios, comunicación pública local cultura popular, Cumanayagua.

\section{Abstract}

Studies of mediation in Latin America have

gained greater booms in recent decades,

through of the reflections analytical around

of public media, community and local. His

approach has been characterized by being

multidisciplinary, various Social Sciences

with the participation, such as Sociology,

Psychology and the Communicology. In

this research are presented somenotes

and reflections on mediations production

from communication local public from

Cumanayagua and its effect processes

socialization and human behaviors in public

spaces, for which we resort to budgets

Manuel M Serrano $(1977,1985,2008)$ Jesús

Martín Barbero $(1987,1995)$ and Guillermo

Orozco (1991).

Keywords

Mediation, mass media, communication local

public, popular culture, Cumanayagua. 


\section{Introducción}

Los medios de comunicación pública, enmarcados en determinado contexto local, no solo deben ser analizados como simples espacios de producción de información comunitaria, sino que las formas y modos en que la comunicación mediática se prolas formas y modos en que la comunicación mediática se procial, donde las mediaciones socioculturales adquieren un valor más preponderante en relación con los medios de las grandes ciudades, demanda de reflexiones ajustadas a tales variaciones y particularidades.

Mientras que en las capitales y otros escenarios mucho más urbanizados se vive cada minuto del desarrollo tecnológico como si fuese el último, donde el contacto permanente con los recursos y dispositivos de máxima categoría, así como el acceso y navegación por las ofertas de la Internet se hacen más axiomáticos, en los contextos locales, se produce y reproduce la socialidad a través del uso de los espacios públicos. De igual manera sucede donde los medios comunitarios y alternativos expresan más compenetración y afinidad con sus públicos estratégicos.

En la esfera de la comunicación pública, la aparición de medios locales deviene en los llamados medios de comunicación pública local, atenuados con el espacio social donde se encuentran y en correspondencia con la misión y la visión proyectada. Algunos ejemplos en Latinoamérica son los periódicos, la radio y la televisión comunitaria. En Cuba, los medios que se localizan en municipios y comunidades son clasificados como locales, donde figuran los boletines, las radios locales y las corresponsalías de TV, anexadas a las llamadas cadenas provinciales que se subordinan al Instituto Cubano de Radio y Televisión (ICRT).

Varias investigaciones desarrolladas por algunos centros de estudio y desde el campo académico de la comunicación en diferentes facultades de Iberoamérica, se han ocupado de realizar análisis y descripciones enfocados en medios locales. En el trabajo, La radio pública local en Andalucía: Una oportunidad para la participación ciudadana, Victoria Gabilondo García del Barco (2013), la autora se ocupa en validar, visibilizar e impulsar el quehacer de la red de radios públicas, realizando un recorrido histórico del crecimiento y de la situación en la que se encuentran las emisoras municipales en Andalucía.
Por su parte, la Facultad de Comunicación de la Universidad de La Habana y otros Centros de Educación Superior de Cuba, aunque insuficiente, también han priorizado el análisis a dichos medios. Tal es el caso de M. Petronila Segura Núñez (2009) en su tesis, Principios metodológicos para el planeamiento estratégico del trabajo periodístico en la emisora Radio Cabaniguán, del municipio tunero de Jobabo; de Neiky Machado López (2009): TV Escambray, Oasis TV y Corralillo Visión ¿el pálpito de la comunidad? Un estudio sobre las características de la televisión comunitaria latinoamerican de tres corresponsalias de television municipal, y por Dagmar Herrera Barreda (2008), La localidad en pantalla. Un estudio sobre el desarrollo de la televisión de cobertura local en Cuba entre sus reflexiones la propia autora apuntó cómo:

Hablar de medios de comunicación a inicios de siglo XXI parece ser, inevitablemente, un camino que conduce a referencias obligadas para los investigadores: globalización, nuevas tecnologías y su impacto en las comunicaciones, enfrentamientos entre lo local y lo global, complementariedad, exclusión, cambios, concentración, expansión; grandes compañías que se fusionan y pequeñas comunidades que potencian el valor de los microespacios: procesos opuestos y complementarios, que coexisten (Barreda, 2008, p. 1).

En Cumanayagua ${ }^{1}$, como caso de estudio particular, el abordaje investigativo de la CMFK Radio Cumanayagua y de la Corresponsalía de TV, desde la carrera de Comunicación Social de la Universidad de Cienfuegos, por lo general, ha estado en función de diseños y propuestas aplicadas, como estrategias de comunicación y diseños de programas y secciones. Por esta razón, hasta la fecha, no se registran análisis comunicológicos que permitan comprender el estado actual de los procesos de producción, análisis de discursos, estudios de audiencia y el

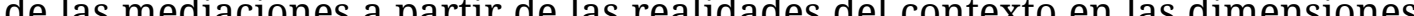
cognitivas y estructurales.

Cumanayagua es un municipio perteneciente a la provincia de Cienfuegos. Se caracteriza por ser eminentemente montañoso y tiene una extensión territorial de $1101.8 \mathrm{~km}^{2}$, de ellos $400.0 \mathrm{~km}^{2}$ corresponden al macizo Guamuhaya. Cuenta con una población de más de 50.000 habitantes. El territorio está compuesto por trece consejos populares, setenta y cuatro asentamiento poblacionales (ocho urbanos y sesenta y seis rurales, de los cuales treinta corresponden al Plan Turquino). El desarrollo cítrico). Entre las empresas más importantes del territorio se destacan: la empresa Cítricos Arimao, la empresa de productos lácteos Escambray, el complejo agroindustrial Eladio Machín y la empresa pecuaria El Tablón. La educación está garantizada en los distintos niveles de enseñanza (primaria, secundaria, preuniversitaria y universitaria). Cuenta con diversas instituciones (CUM), donde se estudian diversas carreras como Agronomía y Contabilidad y Finanzas. Por su parte, recientemente se fundó el Centro de Comunicación para el Desarrollo (CCD), con la misión de incidir desde la comunicación en el mejoramiento humano de Cumanayagua en las dimensiones sociales, económicas y culturales. Uno de los logros impulsados por el país en el territorio es la 作 informativas y culturales de estos. 
El presente trabajo se realiza con el fin de comprender cómo se producen las relaciones (medios de comunicación pública-localmediaciones y contexto) en la localidad de Cumanayagua, usando a la CMFK Radio Cumanayagua y a la Corresponsalía de TV desde sus interacciones con el entorno. Para ello, iniciamos con algunas reflexiones teóricas y conceptuales, a partir de los postulados establecidos en La mediación social y otros aportes de Manuel Martín Serrano (1977, 1985 2008); De los medios a las mediaciones de Jesús Martín Barbero (1987, 1995), y La audiencia frente ala pantalla:una exploración del proceso de recepción televisiva, de Guillermo Orozco (1991). Estos se complementan con autores como Canclini y Piccini (1993), Lameiras y Galindo (1994), Munizaga y Gutiérrez (1983) y otros.

Por último, realizamos una breve descripción de la producción de los medios de comunicación pública local de Cumanayagua y su interdependencia con el sistema social, lo cual permite entender cómo se engendran las mediaciones, y cómo determinadas prácticas sociales de la comunidad desempeñan un rol preponderante en la construcción de la socialidad.

\section{Desarrollo}

\section{Atisbos teóricos}

La teoría de la Mediación social de Manuel Martín Serrano, donde conceptualiza a la mediación "como la actividad que impone limidiacion "como la actividad que impone limites, lo que puede ser dicho, y a las manera de decirlo, por medio de un sistema de orden (Serrano, 1977, p. 76), ha sido atinada para el continente latinoamericano en la década de los ochenta. Esta ofrece una nueva perspectiva estructural para las Ciencias Sociales y, en particular, para la producción y transmisión de la cultura, desde un análisis crítico de odelos culturales y sus funciones en un contexto con detrimento de las democracias re presentativas, militaristas y neoliberales, dependientes de las políticas impuestas por las sociedades occidentales.

En los procesos comunicacionales que tuvieron lugar por esos años en el continente, tal posición teórica contribuyó a que los movimientos populares y sociales percibieran un canal de trabajo posible, a partir de que "la cultura establece estructuras, esquemas, matrices, hábitos y costumbres que se impregnan profundamente en el espíritu de la gente y que esas formas históricas de larga duración, a veces de milenios, otras de siglos, se constituyen en mediaciones fundamentales en los procesos de comunicación humana” (Serrano, 2008, p. 105).

En su obra De los medios a las mediaciones, Jesús Martín Barbero realiza un análisis de la cultura y del fenómeno de la globalización desde una mirada que emana de la semiología. Para esto, hace una interpretación de los medios con sus públicos y de las maneras en que se multiplican los procesos comunicativos. En este sentido, se evidencia cómo a la cultura "hay que pensarla y comprenderla desde las mediaciones" (Lameiras y Galindo, 1994, p. 31). Dichas investigaciones explican el ethos latinoamericano entre lo masivo y lo popular, lo cual permite nuevas lecturas, análisis, reflexiones y críticas, al mirar los mass media en su contexto más amplio.

Otros estudios de Martín Barbero sobre Mediaciones urbanas y nuevos escenarios de comunicación señalan actuales imbricaciones entre la cultura y la comunicación, "[...] no solo a los efectos de los medios y sus innovaciones tecnológicas, sino a las nuevas formas de sociabilidad con los que la gente enfrenta la heterogeneidad simbólica [...] y los modos de experimentar la pertinencia al territorio y las formas de vivir la identidad" (Martín Barbero, 1995, p. 35-47). Tales aportaciones enriquecen lo establecido por Serrano, a partir de la comprensión donde la comunicación se hace cuestión de cultura.

Su tesis circunscribe la configuración de las redes y de los procesos inclusivos que proponen nuevas interpretaciones en la postmodernidad, donde "[...] pensar la ciudad y sus culturas desde la comunicación, entendida como los nuevos modos de estar juntos" (Martín Barbero, 1995, p. 35-47), ofrece una visión diferente para entender este fenómeno, en el cual, los medios de comunicación pública desempeñan un rol preponderante.

A criterio de Munizaga y Gutiérrez, en el ensayo Radio y cultura popular de masas, se establece que la radio facilita enlaces entre las matrices expresivo-simbólicas del mundo con factores racionales de la información instrumental de los espacios urbanizados y modernos (Munizaga y Gutiérrez, 1983).

Al referirse sobre el valor de la radio como "enlace", Matos ha planteado que "la radio ha convocado y mediado dispositivos de enlace de lo territorial con lo discursivo que hacen posible construir espacios de identificación étnica y regional que no son mera expresión de nostalgia de lo local y campesino, sino producción de nuevas formas de socialidad" (Matos, 1988, p. 57).

Con la televisión ocurren formas distintas de percepción, analizadas como fragmentación y flujo, donde la experiencia constituye un elemento superior en relación con el relato televisivo "La televisión convierte al espacio doméstico en territorio virtual”, lo que consti- tuye una reconfiguración de las relaciones privadas y públicas y la superposición de ambos espacios; de ese modo, las audiencias crean sus propias maneras de ver las realidades y presuponen nuevas formas de sensorium ${ }^{2}$. Al respecto, García Canclini, en sus estudios sobre cultura popular, ha planteado "que si las nuevas condiciones de vida en la ciudad exigen la reinvención de los lazos sociales y culturales, son a su vez, las nuevas redes audiovisuales las que efectúan desde su propia lógica una nueva diagramación de los espacios e intercambios urbanos” (Canclini y Piccini, 1993, s.p).

Por tanto, la radio y la televisión son medios de comunicación pública que establecen vínculos culturales en una sociedad, país, provincia o comunidad determinada, lo que induce la necesidad de enfoques multi y transdisciplinares derivados de las aportaciones de la Comunicología y otras Ciencias Sociales ocupadas en afectaciones y procesos similares.

Las prácticas comunicativas y los movimientos que ocurren en las ciudades, reflejan la esencia de los enfoques sobre mediaciones en los estudios latinoamericanos. Asumir estos presupuestos, conscientes de que la envergadura cultural de los medios "[...] no se haya en la cultura-contenido que difunden, sino en el cambio cultural que ellos catalizan y potencian” (Martín Barbero, 1995, p. 35-47), es conocer el valor real de transformación y producción social de las mediaciones.

Para el investigador Guillermo Orozco, en el ensayo La audiencia frente a la pantalla: una exploración del proceso de recepción televisiva, “[...] la preocupación científica y social por

2 Sensibilidad perceptiva que tiene que ver con los fenómenos 2 Sensibilidad perceptiva que tiene que ver con los fenómenos
sensoriales. Il término ha sido abordado por diferentes
teóricos y estudiosos para explicar el fenómeno de la comunicación y las mediaciones, en 
el papel de la televisión (TV) Los estudios de mediaciones aún son insuficientes, esencialtante desde su inserción en la nías de los cambios tecnológicos, a la renovación y al continuo sociedad en los años cincuen- desarrollo de las sociedades contemporáneas. Quizás por ello ta” (Orozco, 1991, p. 53-63). $\quad$ es que diversas reflexiones científicas demandan de mayor atención epistemológica desde la Comunicología, al tiempo que se interpreten y analicen paralelamente las particularidades zado investigaciones de base, de determinados fenómenos sociales, aún y cuando diferentes primero, apoyadas en el mo- estudios y ensayos de reflexión teórica parcelados ocupan el delo de usos y gratificaciones, y posteriormente, desde la y posteriormente, desde la Teoría de la Mediación Social Según nuestro criterio, para tales investigaciones, el punto de de Serrano, enriquecida por partida lo constituye lo cotidiano, la cultura, la política y un enlos aportes del uso social, re- foque CTS ante el vertiginoso cambio tecnológico impuesto por flejados en De los medios a las sociedades globalizadoras y neoliberales. Dicha reflexión es las mediaciones por Martín coherente con el análisis de Hilda Saladrigas Medina, académiBarbero. No obstante, desde ca cubana, cuando establece, en concordancia con los postulael análisis realizado por Oroz- dos de Serrano, que "los medios constituyen hoy espacios claco sobre los postulados de Se- ves de condensación e intersección de múltiples redes de poder rrano, se ha establecido “[...] y de producción cultural” (Garcés y Saladrigas, 2011, p. 46).

que en el enfoque integral de la recepción y doble dimensión de la TV se asume como un todo inseparable en la interacción con la audiencia" (Orozco, 1991, p. 53-63). All se distinguen mediaciones video-tecnológicas, video-tecnologicas, cognoscitivas, situacionales, institu-
cionales y de referencia; en este punto "[...] la producción de sentidos que realiza la audiencia, depende entonces, de la particular combinación de mediaciones en su proceso de recepción [..]" y añade el prorecepción [...]" y añade el propio autor que "la comunida interpretativa de la audiencia, que es donde la audiencia adquiere su identidad como tal, es la resultante de un determinado juego de mediaciones” (Orozco, 1991, p. 53-63). de socialzacio "los medios son agentes culturales y agentes órdenes decón; mediar significa poner en relación distintos órdenes de significación o de experiencia, por ejemplo, la expe(Abril, 1997, p. 110)
En diciembre de 2003, el establecimiento en el municipio de la CMFK Radio Cumanayagua ha posibilitado la socialización de la comunicación y de la información local, provincial, nacional e internacional desde sus variadas ofertas en la programación diaria. Dicha emisora comienza sus transmisiones habituales con el eslogan: Radio Cumanayagua, un sonido propio que llega al corazón de la montaña.

Las transmisiones de la emisora salen al aire por las hondas de los $95.1 \mathrm{MHz}$ y los $105.5 \mathrm{MHz}$, con una potencia de 300 Watts en las frecuencias de FM. Tiene como objetivo central, en correspondencia con las políticas establecidas por el Instituto Cubano de Radio y Televisión (ICRT) "Lograr una producción radial que acerque y satisfaga los gustos, preferencias, acerque y satisfaga los gustos, preferencias, inquietudes y necesidades de la población cumanayagüense y así, contribuir a su enriquecimiento cultural y espiritual, proporcionán2003).

Actualmente, la CMFK cuenta con más de veintinueve programas que salen al aire de domingos a lunes, entre los que se destacan: inforgos a lunes, entre los que se destacan: infor-
mativos, dramatizados, musicales, de crítica periodística, culturales, infantiles y juveniles.

La diversidad de los productos radiofónicos está dada por la propia capacidad de tiempo con que cuenta la institución para producir, destacándose los siguientes ejemplos por clasificación de género:

- Programas musicales monotemáticos: Así es mi tierra, Corazón mexicano, Solo boleros, Momentos, Sabor cubano, Discoteca del domingo, Impacto, La descarga.
Revistas variadas: Con signo de más, Tiempo de sol, Domingo en familia, la revista cultural Espacio abierto y la revista informativa Sin fronteras.

Programa infantil: Amanecer con Andarina y Despierta la Fantasía.

Programa de facilitación social: A buena hora.

Programa de orientación a la mujer y la familia: Con toda idea.

Programa de opinión y debate: Sobre el tapete y Cómo amanece Cumanayagua.

Programa de variedades: Muy cerca de ti y El tiempo pasa.

Programa deportivo: Protagonistas.

- Programa destinado al Turquino: Entre ríos y montañas.

- Programa histórico: De mano con la historia.

- Dos noticieros: uno diario Cumanayagua al día y el dominical En síntesis.

Otros programas que se incluyen en etapa de verano son: el deportivo, Verano olímpico; los a fantasía, y otros la fantasia, y otos musicales como. Ajiaco de verano, Ritmos del Caribe; Siempre dos; Con voz de adentro; Lo que suena el domingo, y Buenos días América. Algunos se especializan por temas o géneros, tal es el caso de Corazón Mexicano; Sabor Cubano; Solo Boleros; Momentos, que difunde la música de las décadas del sesenta y del setenta, y Así es mi tierra, de- 

La interrelación directa que se establece entre la radio en $\mathrm{Cu}$ -
manayagua y las comunidades rurales hasta donde llegan las señales favorece los procesos de comunicación y brinda comseñales favorece los procesos de comunicación y brinda com-
pañía fundamentalmente a aquellas familias que radican en caseríos de la montaña y en los territorios aledaños, propiciando la convivencia armónica, la participación en la solución de las problemáticas y necesidades de los lugareños. Algunos de los radioescuchas entrevistados expresan que la CMFK, además, ha constituido una vía importante para fortalecer las relacio-

Por su parte, la conformación de clubes de aficionados a la radio local, quienes establecen redes de interacción e intercambios por la vía telefónica y en encuentros ocasionales en fechas relacionadas con la celebración de los aniversarios de la radio; así como las relaciones que la CMFK sostiene con las organizaciones locales, las empresas, las instituciones culturales, el sector educativo y la comunidad en general, le otorgan un valor social

De acuerdo con las ideas precedentes, se evidencia cómo los medios locales deben considerar, en primer orden, las expectativas relacionadas con el entorno social, el grado de instrucción o cultural de la población, hábitos de vida, costumbres, hábitos de audiencia, gustos, preferencias y todos los pormenores que nos permitan conocer las particularidades de la región donde está situada la emisora. Es por eso que "las emisoras deben responder a criterios basados en el receptor" (ICRT, 2004, p. 35).

De igual manera, la corresponsalía televisiva: TV Cumanayagua, instalada en el municipio en 2005, hace sus primeras transmisiones con la proyección de imágenes propias del contexto rural y montañoso. Entre la programación que ofrece se destaca el espacio informativo y variado Agenda 7, creado por Diana Merlen Lorenzo, periodista residente en la comunidad.

Esta cuenta con secciones dedicadas a la producción cafetalera, ganadera y citrícola, donde se entrevistan a los protagonistas de las diferentes capas del sector obrero de la localidad. Entre los temas que abordan en el canal, se encuentran los even- tos políticos, sociales, culturales, educativos y medioambientales; donde la crítica periodística se acompaña de logros, retos, cultades que conciernen al desarrollo local, lo que facilita a la audiencia el reflejo de su realidad social mediante la pantalla.

La utilización de tecnologías en el territorio, en función de la producción de comunicación pública, se convierten en formas culturales, a partir de las cuales se promueven nuevos modos de entender la realidad de los aconteceres. Esto origina efectos específicos en el lenguaje mediático local; ello suscita la elaboración de discursos particulares, donde la legitimación de los elementos técnicos, ideológicos y profesionales se involucran en los procesos productivos al servicio de los intereses y necesidades de los pobladores.

Cada medio posee un contenido polisémico, y por ende, facilita distintas maneras de interpretación por la audiencia, pero a la vez definitivo para la captación del mensaje dominante; lo que nos parece atinado en el caso de Cumanayagua, al demostrarse una tendencia elevada de que los significados se reproduzcan en los receptores de manera positiva; aún y cuando todavía hayan zonas de exclusión transmisora y segmentos poblacionales donde se deberá influir con mayor fuerza.

Hablar de mediaciones en un contexto como Cumanayagua, a partir de la producción de comunicación publica local (mediática), demanda sobretodo entender las dimensiones que intervienen en dicho proceso. En el presente esquema se exponen algunas ideas de las cuales parte esta premisa.

Las aportaciones cognoscitivas de estas mediaciones demuestran profundas imbricaciones, las cuales se aprecian desde una perspectiva holística de la recepción y en su interacción, a partir del contenido y el significado. También sucede en gua, dunanaconductas y hábitos sociales en las familias, el amor al terruño y a la patria, y al desarrollo de valores compartidos. El ser humano se ubica como centro, para el cual se ofrece un espacio vital en la continua: familia-escuela-comunidad, de expresión espontánea a tenor con los $\mathrm{XXI}$.

El subsistema económico debe ser analizado de forma particular, teniendo en cuenta las actividades económicas fundamentales de la localidad, destacándose el cultivo del café, de cítricos, la producción agropecuaria y de otros cultivos varios; a este espectro se insertan las empresas, el sector privado y los comercios y establecimientos destinados a prestar servicios públicos.

Las características geográficas constituyen otro de los aspectos influyentes en este sentido, a partir de la delimitación territorial del municipio, compuesta por montaña, premontaña y llanuras. En las serranías cumanayagüenses se ubican tres consejos populares conformados por asentamientos rurales donde residen más de 6525 habitantes, "el diseño sonoro de la emisora parte, fundamentalmente,

del entorno geográfico, un valle entrerriano y un macizo montañoso con una fauna y flora representativas del lugar" (CMFK 2013). Quizás por ello es utilizado diariamente para dar apertura a la programación, el slogan: Radio Cumanayagua: un sonido propio que llega al corazón de la montaña. Y así, cada una de las dimensiones expresadas en el esquema anterior se impregnan en los procesos de producción de los medios locales y al mismo tiempo, los medios se insertan en el conter yecciones ajustadas a tales peculiaridades. 
Algunas de las características que identifican al campesino cumanayagüense se relacionan con las formas y los modos de selección de los productos comunicativos, hasta en la propia ubicación espacial de los medios tecnológicos (radios o televisores). En el estudio preliminar desarrollado desde el Centro de Comunicación para el Desarrollo de Cumanayagua, se pudo apreciar cómo, en determinadas ocasiones en el hogar ${ }^{3}$ de la familia campesina, se sitúa televisor en la habitación principal de la vivienda (la sala), lo que ofrece un lugar preponderante y la posibilidad de brindar compañía a los niños y los ancianos en este tipo de interacción directa con el medio.

Algunas de las costumbres y tradiciones campesinas, como es la colada de café en horas de la madrugac y 7:00AM, son consideradas en la producción mediática local.

El grueso de la población es mayormente campesina y por lo general se levante campesina tan muy temprano en la mañana, es por eso que las transmisiones comienzan las 6:30am todos los días, con el despertar de un gallo para dar entrada a un programa campesino que es amenizado por un grupo musical y repentista de la localidad [ ] La planta es identificada eación de Roberto Novo que caracteriza el entorno, como es la caída de una cascada y el trinar de pájaros (CMFK, 2013).

Pero a este análisis se inserta también un público variado residente en el contexto urbano: desde los niños, para escuchar el progra- ma infantil Amanecer con Andarina, antes de marchar ha en sus quehaceres cotidianos, los trabajadores por cuenta propia, los ancianos en la Casa de Abuelos de la localidad, los vendedores ambulantes, los obreros de la fábrica de tabacos $\mathrm{El}$ Coloso o en el Hogar Materno [...].

Es tradición de este municipio que trabajadores del sector no estatal en sus negocios tengan un radio para escuchar los programas de la CMFK; similar ocurre en el hogar, donde las mujeres acostumbran llevar consigo la radio de un espacio a otro en tiempos de descanso o cuando se realizan labores domésticas, como el lavado de la ropa, barrer los patios, la limpieza del piso y la elaboración de alimentos en la cocina, con el fin de mantenerse atentas a la programación de su interés (musicales, noveas, de facilitación social, deportivos, culturales y otros).

A propósito de los nuevos medios y herramientas derivadas del progresivo desarrollo tecnológico de las últimas décadas, donde figuran la introducción y uso en el país de dispositivos como las memorias USB, los MP3 y MP4, el el DVD, las computadoras y las llamadas tabletas electrónicas, se debe revisar cómo actúan en la población actual en relación con las mediaciones antes referidas, a causa del consumo de productos comunicativos representativos de las sociedades capitalistas, desde materiales que promueven a la violencia, como aquellos que circulan con excesivo contenido erótico.

El estudio realizado permitió identificar cómo estas dificultades se reproducen sobretodo en segmentos poblacionales de adolescentes y jóvenes, y básicamente en el territorio urbano; por lo que una mirada a este grupo etario desde las instituciones educativas, políticas y sociales, así como el control e influencia familiar, ha de ser atinado y pertinente. No ocurre así en zonas rurales como fincas aisladas, caseríos dispersos por la geografía montañosa del Guamuhaya y en escuelas de interno, donde el recurso de la oralidad en los espacios públicos, como en los Círculos Sociales, adquiere una connotación mucho más preponderante.

En cuanto a las mediaciones referenciales, parece notorio que la mayoría de las familias campesinas entran en interacción negativa con los demás miembros del núcleo, cuando los mensajes de diferentes programas abordan temas sensibles, en particular, de género, en relación con la violencia; la convivencia familiar, y la diversidad sexual. En este sentido, los efectos mediadores que proceden de la comunicación pública local aún no adquieren toda la relevancia que les permita discernir el valor de la igualdad y el rechazo a los estereotipos patriarcales, por lo que se puede concluir que el efecto de las mediaciones en el escenario rural y urbano de Cumanayagua indica que aún existen nichos, retos y nuevas perspectivas para el logro de un cambio social aceptable, con el fin de lograr una comunidad interpretativa de las audiencias, con énfasis en valores compartidos desde esta dimensión, donde la producción de medios locales desempeñen un rol protagónico y decisivo, en la consolidación de la tolerancia y la equidad de género.

Las mediaciones posibilitan el conocimiento de nodos de imbricaciones de la cultura popular y tradicional del territorio en relación con los medios, así como las formas de sociabilidad que se han dado desde la apropiación, representación, producción y reproducción social de sus habitantes, y dan algunos modos de experimentar su apego al territorio en la experiencia cotidiana de sus prácticas para vivir la identidad local.

\section{Algunas tendencias locales}

El estudio realizado también permitió identificar algunas tendencias propias del contexto local, que nos aproximan a las singularidades de Cumanayagua, escenario donde los medios y las mediaciones se entretejen y conforman matrices culturales preestablecidas y derivadas, y que impactan en las dificultades anteriormente expresadas:

- Hay una base fuertemente machista entre las familias campesinas alejadas de la ciudad y en determinadas agrupaciones sociales urbanas, quienes practican "juegos tradicionales" peleas de gallos y juegos de dominó, en cualquier momento del día y con apego a la ingestión de bebidas alcohólicas, además de algunos comportamientos que no se adecúan a las tradiciones genuinamente cubanas.

- Existen flujos migratorios o desplazamientos de población rural hacia la ciudad, lo que evidencia una resistencia a distintas mediaciones con las cuales no se identifican por su raíz eminentemente campesina.

Oralidad excesivamente grotesca en determinados espacios públicos, donde se tejen gramáticas y nuevos modos de habitar (colas en bodegas, paradas de transporte, terminales de ómnibus, el Prado, la Glorieta, la Plaza y en coches de tracción animal), desde los cuales se escuchan frases de saludo y contestación como: idime hierro!, ¡ icómo 
estás fiera!, ¡tropa!, ¡ahí, en la lucha!, y ¡qué bola!; que en muchas ocasiones, que bolat, que en, muches tienden a ser calificadas como parte de la vulgaridad, lo que reviste significación desde la perspectiva de las mediaciones estructurales y cognitivas y las dinámicas productivas de los medios locales.

Ello no significa que asumamos una postura esquemática o crítica en extremo, sino que frases como estas, fritos cóno estas, ajustadas a registros en ámbitos más interpersonales, se consideran como adecuadas para tales situaciones de comunicación. La irregularidad se evidencia cuando se van de control y se transportan a espacios donde no son asumidas como normales y adecuadas (en espacios públicos y en instituciones locales).

Los temas de referencia de los medios de comunicación de masas, en cuanto se ocupan de dar cuenta del acontecer, se originan en las transformaciones de entorno social. Por esta razón el cambio social compromete el modo de hacer de los medios, al margen de que cada uno de ellos se comprometa tomando partido a favor o en contra de determinadas transformaciones socio-políticas (Serrano, 1986, p.133)

En Cumanayagua, como caso de estudio particular, es evidente la necesidad de redimensionar el contenido y significado de algunos programas mediante monitoreo e investigaciones sociales de audiencias, sobre todo en aquello sectores de resistencia, en correndencia con el papel decisivo y preponderante que otorgamos, asi como las instituciones estatales (ICRT) que dirigen estas actividades en el pais, toda vez que aún persisten zonas de silencio donde las señales transmisoras no llegan. Concordamos también con Martín Barbero, cuando deja por sentado que:
Más que de medios, la comunicación se nos hace hoy cuestión de mediaciones, esto es de cul nto necesitada no solo de conocimientos, sino de reconocimiento. Un reconocimiento que es, en primer lugar, desplazamiento metodológico para prever el proceso entero de la comunicación desde su otro lado: el de las resistencias y las significaciones que se ejercen desde la significaciones ctividad de apropiación desde los usos que los diferentes grupos sociales, clases, etnias, generaciones, sexos hacen de los medios y los productos masivos. $\mathrm{Y}$ en segundo lugar, reconocimiento histórico: reapropiación histórica del tiempo de la modernidad latinoamericana (Martín Barbero, 1985, p. 3).

Cualquier análisis que se realice desde la comunicología u otra ciencia social a la producción de comunicación pública (mediática) y específicamente en los ámbitos locales, requiere de una mirada mucho más profunda y diversa que si se tratase del estudio de los grandes medios de comunicación masiva.

En el caso específico de Cumanayagua, para entender los procesos de interdependencia entre el subsistema mediático y el sistema social (local), en relación con las mediaciones a los que ambos están sujetos, debemos partir de la propia condición en que se produce la socialidad, del carácter articulador y conformador de las redes de intercambio y de interacción, donde, evidentemente, cualquier tipo de espacio privado (el ámbito familiar) y público (el Prado, la Plaza, los mercados, la Glorieta y el Parque) son capaces de reproducir procesos interactivos generados por los sujetos sociales en sus relaciones cotidianas e históricas.

Quizás por ello, a pesar de los usos inadecuados que muchos dan a las tecnologías, al tra- tarse de un ámbito específico concreto, factores como la extensión territorial de la comunidad y por las dinámicas donde se construye lo público locomuníla lo popular y lo emergente, tomar sentido con mucha más naturalidad. A diferencia de cómo ocurren estos procesos en las grandes ciuda dios de comuniosos en las grandes ciudades, ello hace que los medios de comunicación pública local, de conjunto con los espacios públicos y las prácticas culturales desarrolladas en ellos, desempeñen un papel preponderante en el desarrollo y perpetuación de la comunidad.

\section{Conclusiones}

En relación con la producción de comunicación pública local, la investigación constituyó una aproximación al estado actual de las mediaciones en Cumanayagua, destacándose el desempeño de la CMFK Radio Cumanayagua y la Corresponsalía local TV Cumanayagua.

Se expresaron los nexos existentes entre los procesos socioculturales del entorno y las dinámicas productivas de los medios locales.

Se evidenció la necesidad de una nueva interpretación sobre los medios de comunicación pública local y de las mediaciones en el municipio de Cumanayagua, a partir de las características del contexto y las diversas formas de producción y reproducción simbólicas.

Los estudios de mediaciones deben ser analizados de modo integrador, donde se valoren las tendencias positivas y negativas de cada espacio social, así como las perspectivas de intervención comunitaria en función del desarrollo de los procesos comunicativos y la cultura local.

A otros expertos en el tema, se sugiere dar continuidad a esta mirada preliminar e insertar, en sus análisis, reflexiones en torno a cómo se producen las mediaciones a partir del uso de los dispositivos tecnológicos (DVD, MP3, teléfonos celulares, computadores portátiles...) y el consumo de determinadas producciones de la industria cultural. 


\section{Referencias}

Abril, G. (1997). Teoría general de la información. Madrid: Ediciones Cátedra.

Matos, C. (1988). “Radios y públicos populares”. En: Diálogos dela comunicación, no, 19. Lima.

Garcés, E y Saladrigas M, H. (2011). "La categoría mediaciones en los análisis de las lógicas de producción periodísticas en la web”. Mediaciones\Sociales. Revista de Ciencias Sociales y de la Comunicación, no 9, pp. 35-72. DOI: 10.5209/rev-MESO.2011.n9.38010;http://dx.doi. org/10.5209/rev-MESO.2011.n9.38010.

García, C y Piccini, M. (1993). Cultura de la ciudad de México: símbolos colectivos y uso del espacio urbano. En: El consumo Cultural en México. México: Calcuta.

García Del Barco, G. (2013). "La radio pública local en Andalucía: Una oportunidad para la participación ciudadana”. En: Historia y Comunicación Social, Vol. 18. № Esp. Nov, pp. 257-270.

Grandi, R. (1995). Textos y Contexto en los medios de Comunicación. Barcelona: Bosh

ICRT. (2004). Manual de Control de Calidad. Programación Radial. La Habana: Dirección y Planeamiento de la Radio Cubana.

Jacks, N. (1994). Televisión e identidad en los estudios de recepción. En: Orozco, G. (coord): Televidencia; perspectivas para el análisis de los procesos de recepción televisiva. México: Universidad Iberoamericana.

Lameiras, J. y Galindo, C. (1994). "Medios y mediaciones: los cambiantes sentidos de la dominación en México”. Tlaquepaque, Jal. ITESO / El Colegio de Michoacán.

Martín, J. (1987). "La comunicación desde la cultura. Crisis de lo nacional y emergencia de lo popular, Alternatividad Latinoamericana”, Comunicação e Sociedade, nº 6, pp. 42-50.

Martín, J. (1987). De los medios a las mediaciones. Comunicación, cultura y hegemonía Barcelona: Gustavo Gili.

Martín, J. (1995). "Mediaciones urbanas y nuevos escenarios de comunicación”. En Sociedad, no. 5, octubre. Buenos Aires.

Martín, S, M. (2008). La mediación social. Madrid: Akal.

Martín, S, M. (2001). La Producción Social de Comunicación. Madrid: Alianza Editorial.

Martín, S, M. (1991). Teoría de la Comunicación: Epistemología y análisis de la referencia. México: UNAM/ENEPA.

Martín, S, M. (1985). La mediación de los medios de comunicación de masas. En Moragas, M (ed.). Sociología de la comunicación de masas. Barcelona: Gustavo Gili.
Martín, S, M. . (2007). Teoría de la comunicación. La comunicación, la vida y la sociedad. Madrid: McGraw-Hill.

Quail, D. (1991). Introducción a la Teoría de la Comunicación de masas. Barcelona: Paidós.

Mestre, J. (2007). "Los medios de comunicación y el proceso de aprendizaje”. Recuperado de: http://www.aldeaglobal.net, consultado el 5 de noviembre de 2014.

Munizaga, G. y Gutiérrez, P. (1983). Radio y cultura popular de masas. Santiago: Seneca

Orozco, G. (1991). "La audiencia frente a la pantalla. Una exploración del proceso de recepción televisiva”, Diálogos de la Comunicación, Revista de la Federación Latinoamericana de asociaciones de Facultades de Comunicación Social (FELAFACS) \#30, Lima, junio de 1991, pp. 53-63.

Portal, M. y Trujillo, H. (2008). Comunicación y Sociedad cubana. La Habana: Félix Varela.

Sánchez, R. (2002). "La investigación latinoamericana de la comunicación y su entorno social: notas para una agenda”. En: Diálogos de la Comunicación, nº 64, pp.25-36.

Sztompka, P. (1995). Sociología del cambio social. Madrid: Alianza Editorial

Thompson, J. (1993). Ideología y cultura moderna. Teoría crítica social en la era de la comunicación de masas. México: UNAM.

Vasallo de Lópes, M. (1995) “Recepción de medios, clases, poder y estructura: cuestiones teórico-metodológicas de investigación cualitativa de la audiencia de los medios de comunicación de masas”. En Comunicación y Sociedad, n² 24, pp. 85-96.

Williams, R. (1974). Television, Technology and Cultural Form. Londres: Verso.

Wolf, M. (1987). La investigación de la comunicación de masas. Barcelona: Paidós. 